\title{
Clinical Significance of Rab27a as a Urinary Biomarker in Patients With Bladder Cancer
}

\author{
Ja Yoon $\mathrm{Ku}^{1,2}$, Eu Chang Hwang ${ }^{3}$, Chan Ho Lee ${ }^{4}, \mathrm{Kyung} \mathrm{Hwan} \mathrm{Kim}^{5}$, Dong Deuk Kwon ${ }^{3}$, \\ Hong Koo $\mathrm{Ha}^{2,5}$ \\ 'Department of Urology, Dongnam Institite of Radiological \& Medical Sciences, Busan, Korea \\ ${ }^{2}$ Biomedical Research Institute, Pusan National University Hospital, Busan, Korea \\ ${ }^{3}$ Department of Urology, Chonnam National University Medical School, Gwangju, Korea \\ ${ }^{4}$ Department of Urology, Inje University Busan Paik Hospital, Inje University College of Medicine, Busan, Korea \\ ${ }^{5}$ Department of Urology, Pusan National University Hospital, Pusan National University School of Medicine, Busan, Korea
}

Purpose: The aims of this study were to investigate the clinical value of Rab27a as a urinary biomarker, and its efficiency in the prediction of bladder cancer grade.

Materials and Methods: The expression of Rab27a in urine samples of patients with bladder cancer, cell line (T-24), and tissue samples of patients with bladder cancer was estimated via quantitative reverse transcription polymerase chain reaction (qRT-PCR). The Rab27a expression level was investigated according to sex, age, and histological grade via qRT-PCR and Western blotting.

Results: Rab27a was also expressed at high levels in urine compared to cell lines and tissues from bladder cancer patients. In addition, Rab27a expression varied significantly according to tumor grade $(p<0.001)$. Rab27a was expressed at high levels in male and elderly patients, however, there was not statistically significant.

Conclusions: Our results indicated that Rab27a is valuable as a urinary diagnostic biomarker for bladder cancer. In addition, it may serve as a predictive factor for determining bladder cancer grade.

Key Words: Biomarker, Liquid biopsy, Rab27a, Urinary bladder neoplasms, Urine
Received January 28, 2022

Revised February 4, 2022

Accepted February 8, 2022

Corresponding author:

Dong Deuk Kwon

Email: urokwon@gmail.com

https://orcid.org/0000-0002-1068-3883

Co-corresponding author:

Hong Koo Ha

Email: hongkooha@pusan.ac.kr

https://orcid.org/0000-0002-8240-7765

\section{INTRODUCTION}

Urothelial cancer is the tenth most common cancer in the world, with 549,000 new cases in 2018, an increase from the 430,000 cases reported in 2012. Over $90 \%$ of the urothelial cancers affect the bladder, and the remaining $10 \%$ affect other organs (ureter and renal pelvis). ${ }^{1,2}$ Bladder cancer is often asymptomatic, and is characterized by intermittent hematuria, dysuria, and frequency. These symptoms are similar to those of urinary tract infections. Bladder cancer is mainly detected using cystoscopy and cytology. Cystoscopy detects mass-like lesions; however, it is an invasive endoscopic procedure. ${ }^{3}$ In contrast, cytology shows an acceptable sensitivity and specificity for the detection of high-grade bladder cancer; however, its sensitivity for lowgrade bladder cancer ranges from only $4 \%$ to $31 \%{ }^{4}$ While early detection is important in all cancers, it is especially important in bladder cancer because nonmuscle invasive bladder cancer (NMIBC) and muscle invasive bladder cancer cases vary greatly in their treatment and prognosis. However, most patients are reluctant to undergo cystoscopy 
owing to discomfort, which delays the detection of bladder cancer or may even lead to recurrence.

Rab proteins are part of the Ras superfamily; they are localized to cell membrane compartments. ${ }^{5}$ It has been shown that Rab proteins play an important role in intracellular trafficking and secretion in response to cancer proliferation, migration, and invasion. Among the Rab proteins, Rab27a present in bladder cancer tissues is related to bladder cancer growth and invasion. ${ }^{6}$ However, the clinical significance and efficacy of Rab27a in easily accessible biofluids such as urine have not been investigated. Bladder cancer diagnosis or tumor grade detection using urine, rather than tissue or blood samples, would be of great clinical significance. In this study, we evaluated Rab27a protein levels in the urine samples of patients with bladder cancer, and investigated the efficiency of using Rab27a for the prediction of bladder cancer grade.

\section{MATERIALS AND METHODS}

\section{Patients and Preparation of Urine Samples}

In total, 10 tissue samples and 67 urine samples were obtained from 79 patients who underwent transurethral resection of bladder tumor (TURBT). All samples were obtained during the TURBT and were collected following the Institutional Review Board of Pusan National University Hospital (1703023-052) approval; all subjects provided written informed consent according to the Helsinki declaration. Inclusion criteria included patients with histologically confirmed urothelial cancer. Exclusion criteria for sample collection from the patients were as follows: patients who underwent a second TURBT, and patients who were already diagnosed with metastasis or showed higher than stage cT3 cancer. For the initial screening, 30 $\mathrm{mL}$ of voided urine was collected from patients with bladder cancer and from healthy subjects as controls. Samples were collected mostly in the morning in sterile plastic containers and transported in refrigerated conditions to the laboratory as soon as possible. Urine samples were centrifuged at $750 \times \mathrm{g}$ for 10 minutes at $4{ }^{\circ} \mathrm{C}$, to remove the insoluble materials within $30-120$ minutes of collection to prevent protein release from the artifacts. Subsequently, the supernatant after centrifugation was transferred to new preservation tubes (Norgen Biotek, Thorold, ON, Canada) and stored until further experiments; the cell pellets (debris) were discarded.

\section{RNA Extraction From Urine Samples}

Total RNA from urine samples was extracted using the Urine Total RNA Purification Maxi Kit, Slurry Format (Norgen Biotek), as per the manufacturer's standard protocol. In brief, $0.35 \mathrm{~mL}$ of slurry C3 and $4.65 \mathrm{~mL}$ of lysis buffer were added to $5 \mathrm{~mL}$ of urine samples, followed by the addition of $5 \mathrm{~mL}$ of $96 \%$ ethanol. After mixing the solution, the urine mixture was centrifuged for 5 minutes at $2,000 \times \mathrm{g}$ and the supernatants were discarded. The wash solution $(500 \mu \mathrm{L})$ was then added to the pellet and mixed, after which the solution was transferred to a mini filter column. The column was washed twice with the wash solution, after which $500 \mu \mathrm{L}$ of 96\% ethanol was added, followed by centrifugation, and then the filtrate was discarded. Finally, the elution buffer was added to the dried mini spin column and centrifuged to obtain the purified RNA. The RNA concentrations were determined using the Gen5 2.01 software of the Synergy H1 Hybrid MultiMode Microplate Reader (BioTek, AHSI, Milan, Italy).

\section{Reverse Transcription (cDNA Synthesis)}

The isolated RNA was converted to first-strand 
cDNA using the BioRad iScript cDNA synthesis kit (Bio-Rad, Hercules, CA, USA). As the concentration of RNA extracted from the urine samples was comparatively lower than that isolated from tissues or cells, a total of 100 ng of RNA (equal for each sample) was used for reverse transcription in a final reaction volume of $20 \mu \mathrm{L}$. To minimize experimental variations, cDNA was synthesized concurrently for all samples, and was stored at $-20^{\circ} \mathrm{C}$ until use.

\section{Quantitative Reverse Transcription Polymerase Chain Reaction}

Quantitative reverse transcription polymerase chain reaction (qRT-PCR) was performed using a FastStart Universal SYBR Green Master mix (Roche, Basel, Switzerland) in an Applied Bioscience 7500 thermocycler (Thermo Fisher, Waltham, MA, USA). The reaction mix was subjected to denaturation at $95^{\circ} \mathrm{C}$ for 10 minutes, followed by 40 cycles of denaturation at $95^{\circ} \mathrm{C}$ for 15 seconds and annealing at $58^{\circ} \mathrm{C}$ for 1 minute. The primer sequences used for the genes were: Rab27a, sense 5'-GAAGAC CAGAGGGCAGTGAA-3', and antisense 5'-ACTGGT TTCAAAATAGGGGATTC-3'; $\beta$-actin, sense 5'-AGA GCTACGAGCTGCCTGAC-3', and antisense 5'AGCACTGTGTTGGCGTACAG-3'. Expression levels of $R A B 27 A$ were normalized to those of $\beta$-actin (endogenous control).

\section{Western Blotting}

Western blotting was used to analyze the urine samples after they were centrifuged to exclude cells. Protein concentrations were assessed using the bicinchoninic Protein Assay Kit (Pierce, Rockford, IL, USA). Proteins (150 $\mu$ g) from individual samples were resolved using sodium dodecyl sulphate-polyacrylamide gel electrophoresis, and transferred to polyvinylidene difluoride membranes (Bio-Rad). The membranes were then blocked with
$5 \%$ bovine serum albumin in tris-buffered saline containing $0.1 \%$ Tween-20 for 1.5 hours at room temperature, followed by probing with primary RAB27A polyclonal antibodies (ABClonal, Woburn, MA, USA). Primary and secondary antibodies were diluted in the same blocking solution. The bound secondary antibodies were detected via enhanced chemiluminescence (ECL) using the Pierce ECL Western Blotting Substrate (Thermo Fisher), and using a ChemiDoc XRS+ imaging system (Bio-Rad, Feldkirchen, Germany).

\section{Statistical Analysis}

For each sample and target, the mean, standard deviation, and percent coefficient of variation (standard deviation/mean $\times 100$ ) were calculated. Band intensity from the Western blots was quantified using the ImageJ program (National Institutes of Health, Bethesda, MD, USA). A p-value of $<0.05$ (2-sided) was considered significant.

\section{RESULTS}

We investigated Rab27a expression in 10 tissue samples and 67 urine samples using qRT-PCR. The expression of Rab27a in urine samples of bladder cancer patients according to clinical pathology characteristics is shown in Table 1 . As shown in Table 1, a significant difference was found between low-grade and high-grade tumors. In low-grade tumors, the mean value of Rab27a expression was $1,592.1 \pm 3,105.7$, and the mean expression value in high-grade tumors was $76.1 \pm 186.8$.

\section{Correlation of Rab27a Expression in Tissue and Urine Samples in Patients With Bladder Cancer}

The expression of Rab27a protein was investigated in normal urothelial and bladder cancer tissues for comparative analysis. Immunostaining 
Table 1. Arrangement of Rab27A in bladder cancer urine according to clinic-pathological characteristics

\begin{tabular}{lccc}
\hline Characteristic & No. of patients & Mean of Rab27A expression & p-value \\
\hline Age $($ yr) & 21 & & 0.119 \\
$61-70$ & 38 & $215.7 \pm 403.6$ & \\
$71-80$ & 8 & $1523.8 \pm 3562.9$ & 0.209 \\
$81-90$ & & & \\
Sex & 54 & $574.8 \pm 1962.8$ & 0.048 \\
Male & 13 & $220.3 \pm 294.5$ & \\
Female & & & \\
Tumor grade & 19 & $7592.1 \pm 3105.7$ & \\
Low grade & 48 & $76.1 \pm 186.8$ & \\
High grade &
\end{tabular}

Values are presented as mean \pm standard deviation.

A

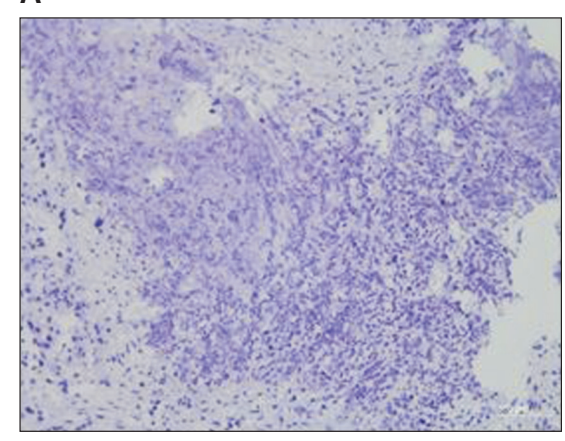

B

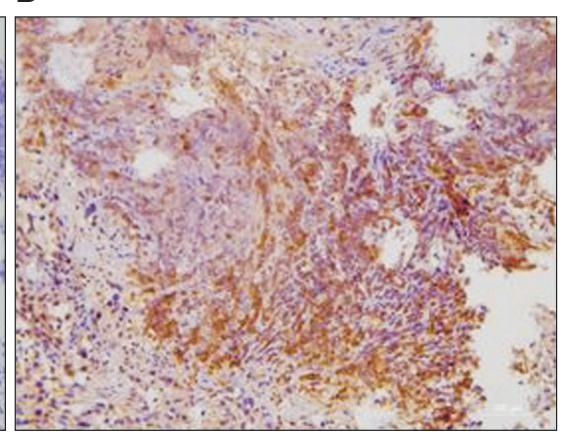

C

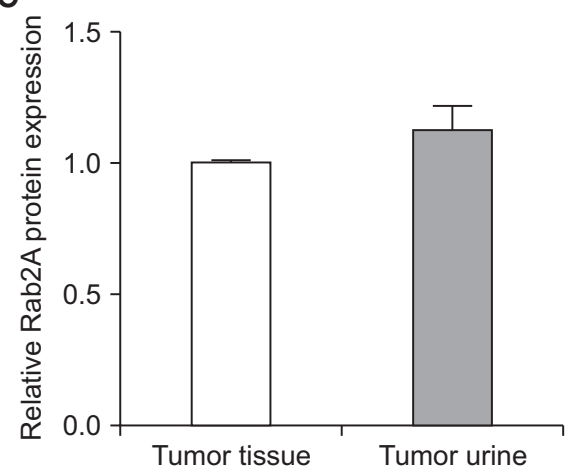

Fig. 1. Expression of Rab27a in bladder cancer tissue and urine. (A) Negative Rab27a staining in the normal bladder tissue. (B) Positive Rab27a in the muscle invasive bladder cancer tissue. (C) Rab27a protein expression was examined in 10 paired tumor tissue and urine. Relative staining intensitu was quantified by GraphPad Prism 8.4.0.

revealed absence of Rab27a expression in normal urothelial tissues and presence of Rab27a in bladder cancer tissues (Fig. 1A, B). We compared and analyzed Rab27a expression in 10 paired tissue and urine samples from patients with bladder cancer using RT-PCR (Fig. 1C). There was no significant difference between the 2 groups.

\section{High Rab27a Expression Predicted Low-Grade Tumors}

The optimal cutoff value of Rab27a for predicting tumor grade was 86.6 according to the receiver operating characteristic (ROC) curve using the maximal Youden index (area under ROC $=0.893$; sensitivity, 89.6\%; specificity, 78.4\%) (Fig. 2).

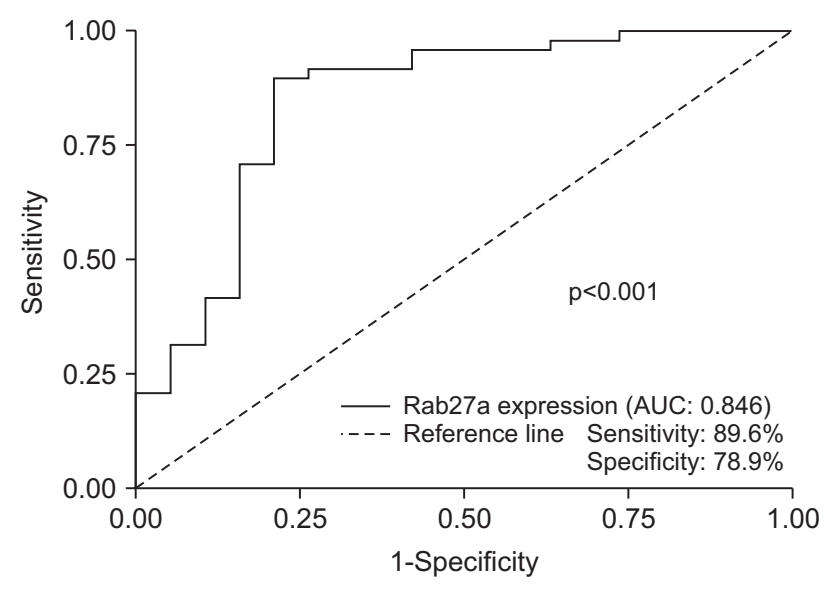

Fig. 2. Receiver operating characteristic curve for Rab27a for predicting tumor grade in patients with bladder cancer. AUC, area under the curve.

Thereafter, all subjects were divided into 2 groups as follows: the low Rab27A (Rab27a expression $\leq$ 
86.6) and high Rab27A (Rab27a expression >86.6) groups. When Rab27a expression $>86.6$ was used as a cutoff, we found 15 patients with low-grade cancer, and 6 patient with high-grade cancer, whereas, when a cutoff of Rab27a expression $\leq 86.6,4$ patients with low-grade cancer, and 42 patients with high-grade cancer were identified. Using the chisquare test, a significant association $(\mathrm{p}<0.001)$ was found between Rab27a expression and tumor grade
(Table 2, Fig. 3). However, analysis of Western blot data revealed no significant correlation between Rab27a expression and tumor grade (Fig. 4).

\section{DISCUSSION}

The role of the Rab27 protein has been reported in several human cancers. Humans contain more than 60 members of the Rab family, each of which

Table 2. Arrangement of Rab27A in bladder cancer urine according to tumor grade

\begin{tabular}{ccc}
\hline & \multicolumn{2}{c}{ Tumor grade } \\
\cline { 2 - 3 } Rab27A expression & Low grade $(\mathrm{N}=19)$ & High grade $(\mathrm{N}=48)$ \\
\hline Rab27A expression $>86.6$ & $15(78.9)$ & $6(12.5)$ \\
Rab27A expression $\leq 86.6$ & $4(21.1)$ & $42(87.5)$ \\
\hline
\end{tabular}

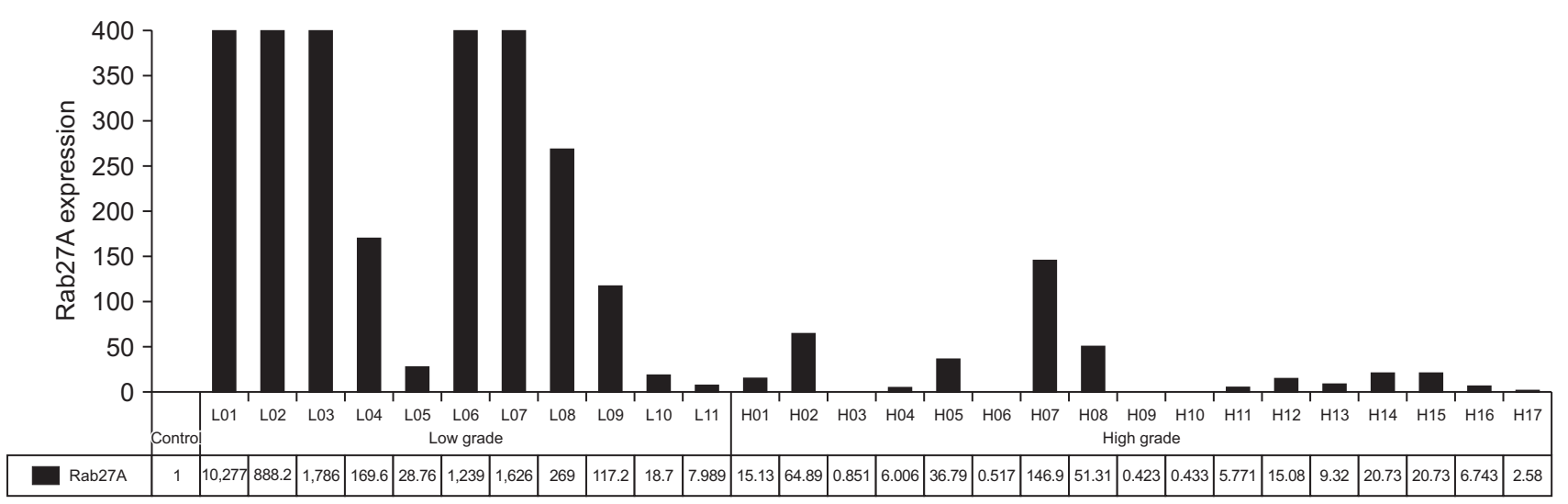

Fig. 3. Expression of Rab27a according to tumor grade by reverse transcription polymerase chain reaction.

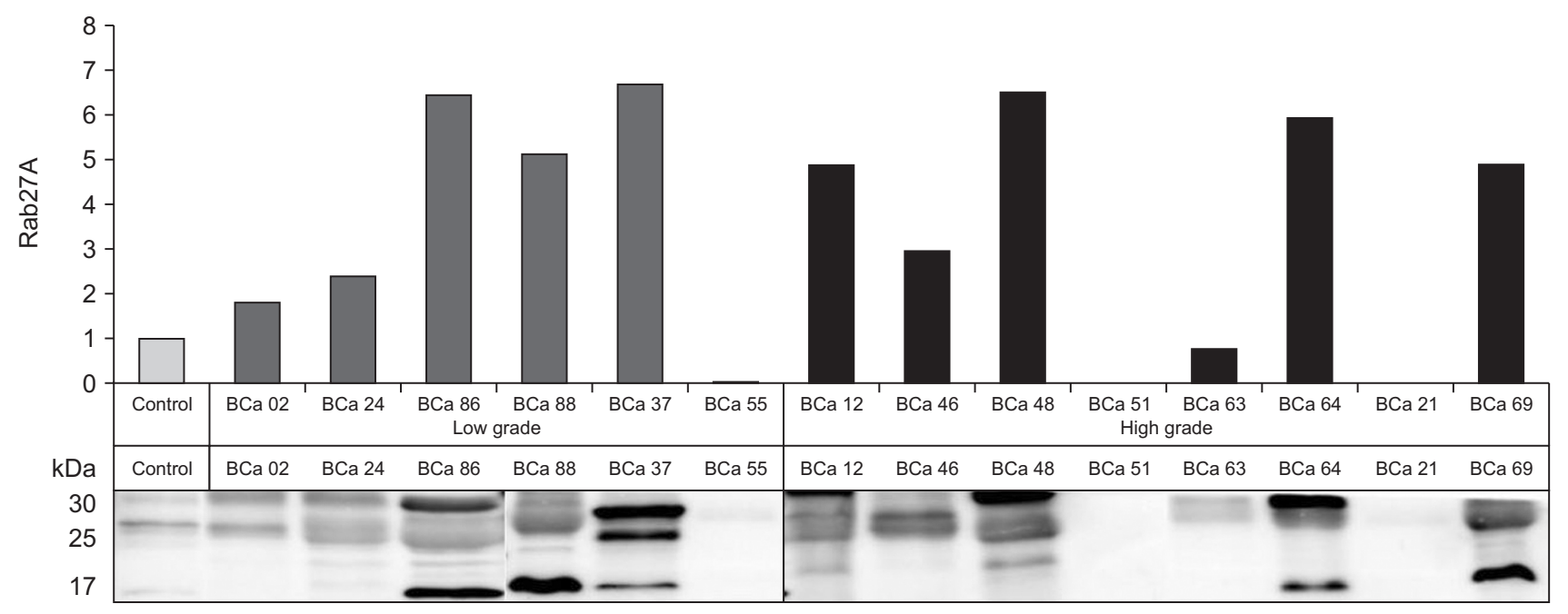

Fig. 4. Expression of Rab27a according to tumor grade by WesternBlot. 
is localized to a specific intracellular membrane compartment and controls the intracellular traffic. ${ }^{7}$ Rab proteins circulate between inactive cytoplasmic GDP-binding forms and active membrane-related GTP-binding forms. In the GTP-binding form, the Rab proteins recruit effector proteins that mediate vesicle metabolism in donor cell organelles, transport microtubules or actin filaments, and are involved in tracking and fusion into receptor compartments. The functions of Rab proteins in tumor cells include the following: transmission and recycling of integrin during cell migration; protease secretion that breaks down the extracellular matrix and promotes invasion of the surrounding tissues; exosome secretion that mediates communication with epileptic cells; and trafficking and control of receptors.

Recent studies have shown that Rab27a contributes to the tumor-stromal cell communication, and modification of the tumor microenvironment; however, whether it acts as a tumor oncogene or suppressor is a controversial subject. Rab27a has been shown to promote invasion during melanoma progression. Rab27a inhibition was found to reduce primary tumor growth and lung metastasis. ${ }^{8,9}$ Rab27a promotes the stemness of colon cancer cells via upregulation of cytokine secretion. ${ }^{10}$ Overexpression of Rab27a is associated with the progression of lymph node metastatic breast cancer. ${ }^{11,12}$ In these studies, Rab27a has been considered as a potential oncogenic protein in human cancer. However, according to several reports, Rab27a may act as a tumor suppressor. A high expression of Rab27a indicates a good prognosis in colon cancer cases. ${ }^{13}$ RAB27A and $\mathrm{RAB} 27 \mathrm{~B}$ are downregulated in advanced prostate cancers. ${ }^{14}$ However, the clinical significance and biological role of human bladder cancer have not yet been identified.

Our results show that lipid biopsy samples (urine) could be used for detecting bladder cancer. Further, they also show that there was no significant difference in Rab27a expression in urine samples compared to that in tissue biopsy. Therefore, Rab27a expression may therefore be considered a parameter for diagnosing bladder cancer using a urine test without invasive cystoscopy.

In addition, when NMIBC treatment is performed from $30 \%$ to muscle invasive bladder cancer, active monitoring after NMIBC treatment is required. Therefore, several patients undergo cystoscopy despite their reluctance owing to discomfort (pain, risk of infection, voiding difficulty), as it can be helpful for active monitoring. ${ }^{15,16}$ Second, the grade of bladder cancer can be predicted based on the expression level of Rab27a. In our study, the expression level of Rab27a was investigated from $78.9 \%$ to low grade in patients with expression levels higher than 86.6, and from $87.5 \%$ to high grade in patients with expression levels lower than 86.6. In bladder cancer, the recurrence rate of high-grade cancer is 7\%-80\%, which makes Rab27a a very useful predictive tool.

The limitation of this study is the small number of samples analyzed. However, we believe that this study has great significance in the diagnosis of cancer using urine, and not tissue samples.

\section{CONCLUSION}

Our study showed that bladder cancer can be diagnosed via the detection of Rab27a in the urine, and that the tumor grade of bladder cancer can be predicted according to expression level. However, further prospective and thorough trials are needed to validate and improve upon these findings.

\section{NOTES}

- Conflicts of interest: No potential conflict of interest relevant to this article was reported.

- Funding: This study was supported by Biomedical 
Research Institute Grant (2017-29), Pusan National University Hospital.

\section{- ORCID}

Ja Yoon Ku: https://orcid.org/0000-0003-3460-9386

Eu Chang Hwang: https://orcid.org/0000-0002-2031-124X

Chan Ho Lee: https://orcid.org/0000-0002-7750-6806

Kyung Hwan Kim: https://orcid.org/0000-0001-7162-6527

Dong Deuk Kwon: https://orcid.org/0000-0002-1068-3883

Hong Koo Ha: https://orcid.org/0000-0002-8240-7765

\section{REFERENCES}

1. Bray F, Ferlay J, Soerjomataram I, Siegel RL, Torre LA, Jemal A. Global cancer statistics 2018: GLOBOCAN estimates of incidence and mortality worldwide for 36 cancers in 185 countries. CA Cancer J Clin 2018;68:394-424.

2. Antoni S, Ferlay J, Soerjomataram I, Znaor A, Jemal A, Bray F. Bladder cancer incidence and mortality: a global overview and recent trends. Eur Urol 2017;71:96-108.

3. Gontero P, Oderda M, Altieri V, Bartoletti R, Cai T, Colombo R, et al. Are referral centers for nonmuscle-invasive bladder cancer compliant to EAU guidelines? A report from the vesical antiblastic therapy Italian study. Urol Int 2011;86:19-24.

4. Lotan Y, Roehrborn CG. Sensitivity and specificity of commonly available bladder tumor markers versus cytology: results of a comprehensive literature review and meta-analyses. Urology 2003;61:10918; discussion 18.

5. Recchi C, Seabra MC. Novel functions for Rab GTPases in multiple aspects of tumour progression. Biochem Soc Trans 2012;40:1398-403.

6. Liu J, Gong X, Zhu X, Xue D, Liu Y, Wang P. Rab27A overexpression promotes bladder cancer proliferation and chemoresistance through regulation of NFkappaB signaling. Oncotarget 2017;8:75272-83.

7. Diekmann Y, Seixas E, Gouw M, Tavares-Cadete F, Seabra MC, Pereira-Leal JB. Thousands of rab GTPases for the cell biologist. PLoS Comput Biol 2011; 7:e1002217.

8. Akavia UD, Litvin O, Kim J, Sanchez-Garcia F, Kotliar D, Causton HC, et al. An integrated approach to uncover drivers of cancer. Cell 2010;143:100517.

9. Peinado H, Alečković M, Lavotshkin S, Matei I, Costa-Silva B, Moreno-Bueno G, et al. Melanoma exosomes educate bone marrow progenitor cells toward a pro-metastatic phenotype through MET. Nat Med 2012;18:883-91.

10. Feng F, Jiang Y, Lu H, Lu X, Wang S, Wang L, et al. Rab27A mediated by NF-kappaB promotes the stemness of colon cancer cells via up-regulation of cytokine secretion. Oncotarget 2016;7:63342-51.

11. Bobrie A, Krumeich S, Reyal F, Recchi C, Moita LF, Seabra MC, et al. Rab27a supports exosome-dependent and -independent mechanisms that modify the tumor microenvironment and can promote tumor progression. Cancer Res 2012;72:4920-30.

12. Montel V, Huang TY, Mose E, Pestonjamasp K, Tarin D. Expression profiling of primary tumors and matched lymphatic and lung metastases in a xenogeneic breast cancer model. Am J Pathol 2005; 166:1565-79.

13. Shi C, Yang X, Ni Y, Hou N, Xu L, Zhan F, et al. High Rab27A expression indicates favorable prognosis in CRC. Diagn Pathol 2015;10:68.

14. Worst TS, Meyer Y, Gottschalt M, Weis CA, von Hardenberg J, Frank C, et al. RAB27A, RAB27B and VPS36 are downregulated in advanced prostate cancer and show functional relevance in prostate cancer cells. Int J Oncol 2017;50:920-32.

15. Thomas F, Rosario DJ, Rubin N, Goepel JR, Abbod MF, Catto JW. The long-term outcome of treated high-risk nonmuscle-invasive bladder cancer: time to change treatment paradigm? Cancer 2012;118: 5525-34.

16. Thomas F, Noon AP, Rubin N, Goepel JR, Catto JW. Comparative outcomes of primary, recurrent, and progressive high-risk non-muscle-invasive bladder cancer. Eur Urol 2013;63:145-54. 